\title{
Active Guidance for Laser Retinal Surgery with a Handheld Instrument
}

\author{
Brian C. Becker, Student Member, IEEE, Cristina Robles Valdivieso, Joydeep Biswas, Student \\ Member, IEEE, Louis A. Lobes, Jr., and Cameron N. Riviere, Member, IEEE
}

\begin{abstract}
Laser photocoagulation is a standard interventional tool in vitreoretinal surgery. Commonly applied treatments such as grid photocoagulation and panretinal photocoagulation involve the application of hundreds of dotlike laser burns to the retina. In order to enhance the accuracy and reduce the tedium of this procedure, we are developing a robot-assisted technique for retinal laser photocoagulation that includes software for planning patterns of laser burns on a retinal image and uses an active handheld micromanipulator known as Micron in order to apply the pattern of burns to the retina. The paper describes the system and presents preliminary results. In a sample $7 \times 7$ pattern of lesions applied to an artificial surface, the system demonstrated a mean position error of $43 \pm 23 \mu \mathrm{m}$.
\end{abstract}

\section{INTRODUCTION}

$\mathrm{R}$ ETINAL laser photocoagulation is a standard treatment of diabetic retinopathy [1]. Panretinal photocoagulation is a treatment for proliferative diabetic retinopathy in which hundreds of laser burns are applied to the retina in order to retard the growth of new abnormal blood vessels. Laser positioning accuracy is important; for example, inadvertent photocoagulation of a vein can cause vein occlusion [2]. Grid photocoagulation involves similar application of a pattern of laser burns, over a smaller area, and is used to treat macular edema [3]. Focal photocoagulation is a another treatment for macular edema in which leaking blood vessels in the retina are directly targeted in order to seal the leaks; laser positioning accuracy is of obvious importance for efficacy [4].

Positioning accuracy in such procedures may be increased by the use of robotics, and semi-automated systems that can apply multiple-burn patterns can reduce the tedium of these treatments for both patient and physician. Blumenkranz et al. described one such system for semi-automated scanning for multiple burns, using mirrors mounted on a two-axis galvanometric scanner attached to a slit lamp, designed for treatment through the pupil [5]. In order to provide similar

Manuscript received April 7, 2009. This work was supported in part by the American Society for Laser Medicine and Surgery, by the National Institutes of Health (grant no. R01 EB007969), the National Science Foundation (Graduate Research Fellowship), and the ARCS Foundation.

B. C. Becker, J. Biswas, and C. N. Riviere are with the Robotics Institute, Carnegie Mellon University, Pittsburgh, PA 15213 USA (e-mail: briancbecker@cmu.edu; joydeep@cmu.edu; camr@ri.cmu.edu).

C. Robles Valdivieso is with the University of Valladolid, Spain.

L. A. Lobes, Jr., is with the Department of Ophthalmology, University of Pittsburgh Medical Center, Pittsburgh, PA 15213 USA (e-mail: 1lobes@retinapittsburgh.com). benefits during intraocular surgery, we are developing a system for accurate robotically-assisted surgical application of multiple-burn laser patterns.

The robotic platform for development of this scanning system for laser photocoagulation is Micron, a fully handheld active micromanipulator that has been reported previously [6-8]. Micron senses its own motion in six degrees of freedom (6DOF) using a frequency-multiplexed optical tracking scheme [9]. It then controls a 3DOF parallel manipulator built into its handle in order to deflect the tool tip or end-effector, either to compensate for undesired motion such as physiological hand tremor [10] or to actively guide the tip toward a known target [7].

This paper reports initial results from the development of a system that includes software for planning of patterns of laser burns on an image of a patient's retina, followed by application of the planned patterns using visual servoing techniques with Micron.

\section{METHODS}

The system for robot-assisted laser photocoagulation using Micron is shown in Fig. 1 and Fig. 2. The operator can view the workspace through the stereo operating microscope or through a 3D computer display (Trimon ZMM220W, Zalman Tech Co., Ltd., Seoul, Korea) shown at the right of the figure.

The custom optical tracking instrumentation of Micron uses position-sensitive detectors (PSDs) to detect four pulsed

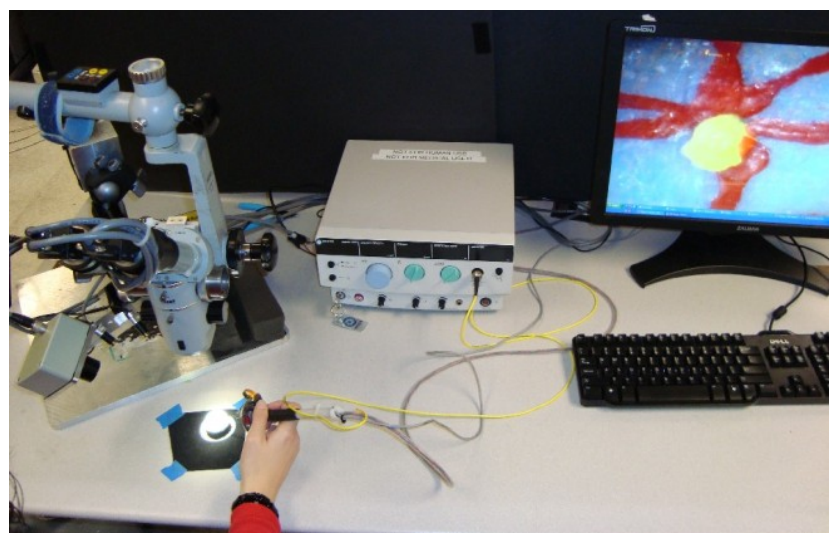

Fig. 1. The laser photocoagulation scanning system setup using Micron, an active handheld micromanipulator. Micron is shown held in the hand of the operator. The operator has the option of viewing the workspace either through the stereo operating microscope (at left) or on a 3D computer display (at right). The laser instrumentation is on the tabletop in the center. 


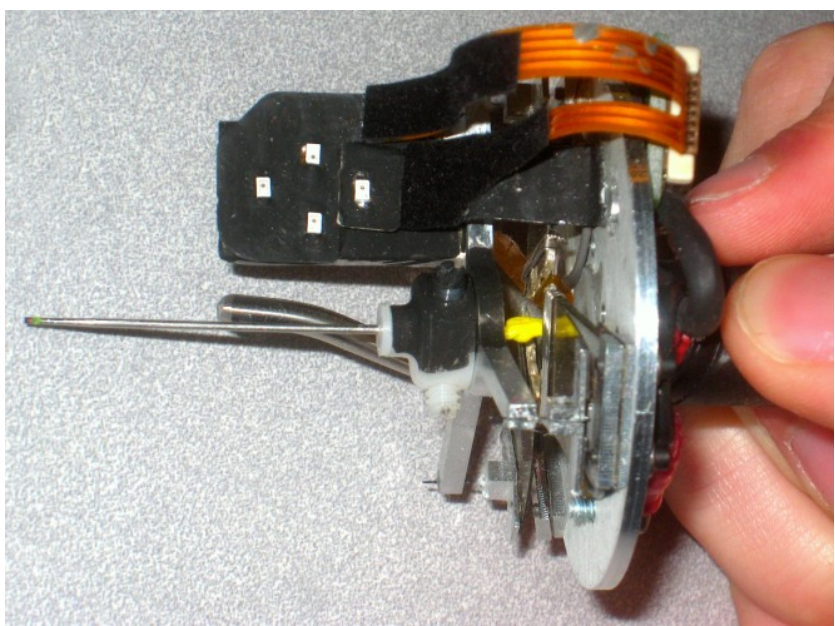

Fig. 2. Close-up view of Micron in the hand of the user. The fiber-optic cable threads through the hollow center of the instrument, running down the actuated metal shaft of the tip. Four white LEDs act as optical trackers to provide micron-accurate positioning of Micron's tip.

LEDs mounted inside small spherical diffusers near the intraocular shaft of the instrument, providing 6DOF tracking of the motion of both the tool tip and the handle [9].

Micron is used under a Zeiss OPMI ${ }^{\circledR} 1$ microscope with $25 \mathrm{x}$ magnification. Two Flea ${ }^{\circledR} 2$ cameras (Point Grey Research, Richmond, BC, Canada), capturing $800 \times 600$ video at $30 \mathrm{~Hz}$, are mounted to the microscope, providing a stereo view of a workspace of roughly $8 \times 6 \mathrm{~mm}$. The tip manipulator of Micron uses Thunder ${ }^{\circledR}$ TH-10R piezoelectric actuators (Face International Corp., Norfolk, Va., USA), and has a range of motion of approximately $0.5 \mathrm{~mm}$ axially and $1.8 \mathrm{~mm}$ transversely.

\section{A. Preoperative Burn Placement}

Planning software for the pattern of dots to be generated by the laser was programmed in LabVIEW ${ }^{\circledR}$ (National Instruments Corp., Austin, Tx., USA). A retinal image is captured through the operating microscope using one of the two tracking cameras. This image is then used as the background upon which the desired pattern can be planned. Fig. 3 shows the graphical user interface of the planning software. The distance between centers of the dots to be

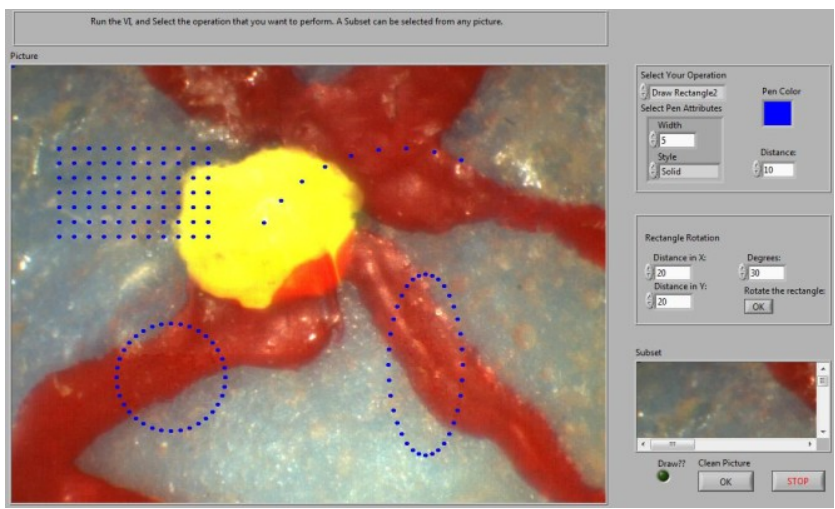

Fig. 3. The graphical user interface of the planning software, showing several of the types of patterns that can be generated. A photograph of the retina (in this case an artificial retina) is used as the background over which a preoperative plan can be made.

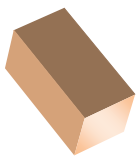

(g)

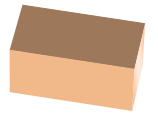

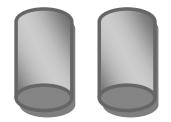

(c) (d) (b)

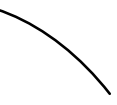

Fig. 4. Micron laser setup: Micron handle (a) attached to laser via fiber optic cable (b). Cameras (c) track the finder beam (d) and target (e) at $30 \mathrm{~Hz}$. Control velocity (f) is calculated in the image and converted to world coordinates used by the positioning sensor $(\mathrm{g})$. The piezoelectric actuators then deflect the tip from position $\left(h_{1}\right)$ to $\left(h_{2}\right)$ to burn target $(e)$.

generated is specified as a selectable parameter in the user interface. A drop-down menu in the graphical user interface allows the surgeon to select a pattern in the shape of a rectangle, circular sector, annulus (or sector of an annulus), circle, or oval. Using any of these primitives, the pattern is positioned and then scaled by clicking and dragging the mouse. Different primitives can be used in succession in order to generate a desired overall pattern. This planning software then generates a file of $x$ and $y$ coordinates of the planned dots, which is sent to the visual control software.

In order to maintain correct intraoperative positioning of the planned pattern on the retina despite possible movement of the eye or head of the patient, the preoperative image used for planning is registered to the real-time image streams of both cameras. Using interest points detected by the Speeded Up Robust Features (SURF) algorithm of Bay et al. [11], a homography is computed with the standard RANSAC algorithm [12] and applied to align the planned pattern to new video frames. This registration is maintained until the entire laser pattern is applied.

\section{B. Control of Micron}

Control of Micron is accomplished using the $x$ and $y$ coordinates of the target dot pattern. Tracked in both stereo frames, the 2D locations of the dots are reconstructed in 3D using multi-view geometry [13]. The color-coded tip of Micron is also tracked with a color-based tracker [14], providing relative positioning between the targets and the tip of the instrument. When in treat mode, a red finder laser serves as guidance for where the laser will burn when activated. The vision system tracks the centroid of the finder beam and generates control signals to move it to the target. A quick $30 \mathrm{~s}$ calibration [7] calculates the transformation between the stereo image pair and the positioning coordinate system, allowing control signals to be generated from these tracked points in the image. The popular Intel OpenCV library is used to implement the computer vision techniques.

Micron uses tremor cancellation during the entire operation for increased positioning accuracy. As the 
operator moves close to a given target, Micron transfers control from its general tremor-canceling mode [8] to a visual-servoing "snap-to" behavior [7], as follows. When in the vicinity of a dot, the control system calculates a control input from the position difference of the target and the location of the finder beam and engages the visual servoing control to move the tip in line with the target.

First, the finder beam location is projected to a local plane containing the target. Second, a proportional controller iteratively reduces the error difference between the target and the finder beam. Finally, once the tip of Micron is pointing at the dot and has stabilized hand motion with the snap-to behavior, the software fires the laser. Control then defaults back to normal tremor compensation. The surgeon can smoothly move to the next dot, which re-engages the snap-to behavior and fires the laser again. See Fig. 4 for a graphical representation of the laser burning control process.

An Iridex Iriderm Diolite 532 Laser with Iridex 25 -gauge endoprobes is used for photocoagulation. Laser pulses of 30 ms duration are used.

\section{RESULTS}

As a preliminary test of the system, Fig. 5 presents the results of a $7 \times 7$ rectangular pattern applied by non-clinical personnel using the system, with spacing of $650 \mu \mathrm{m}$ between burns. Automatic post-processing computer vision algorithms are applied to calculate the centroids of the burns and compare them to the preoperatively designated burn locations. A histogram of error distributions can be found in Fig 6. The mean error in 49 burn placement is $43 \pm 23 \mu \mathrm{m}$. The minimum error over the burns is $6 \mu \mathrm{m}$, and the maximum error is $104 \mu \mathrm{m}$.

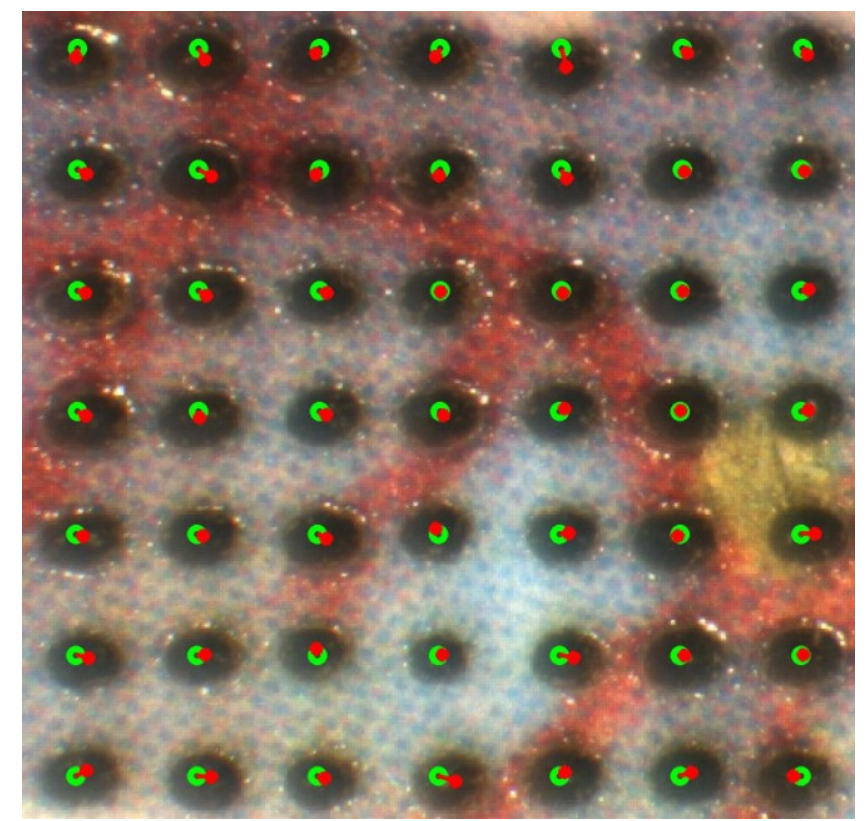

Fig. 5. A sample $7 \times 7$ rectangular pattern generated with the system, with $650-\mu \mathrm{m}$ spacing, on an artificial background (colored to simulate the retina). The automatically detected centroids of the laser burns are marked in red solid circles, and the planned targets are in hollow green circles.

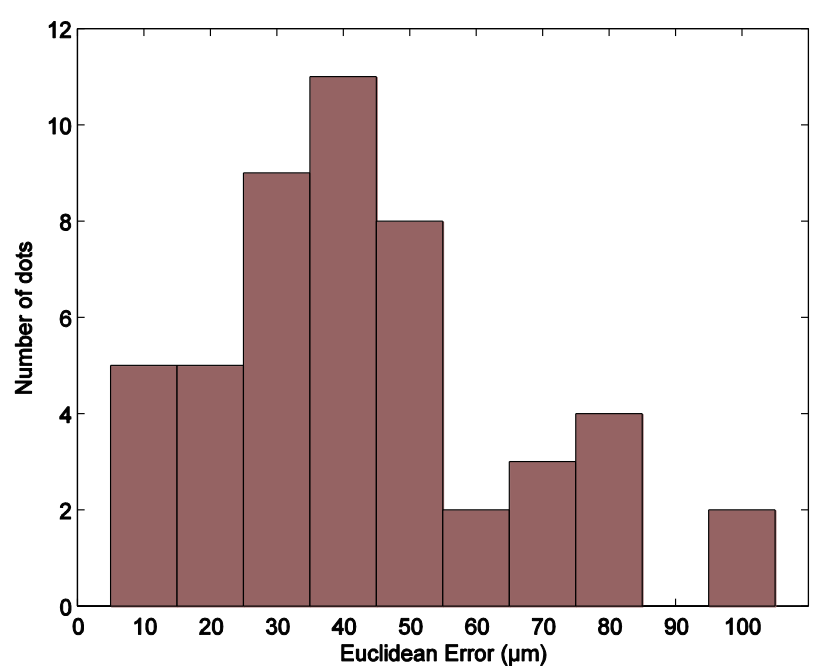

Fig. 6. Histogram of dot placement errors, representing the distribution of distances between desired and actual burn locations.

\section{DISCUSSION}

The results presented demonstrate the basic feasibility of robotically-assisted surgical grid or panretinal laser photocoagulation using an active handheld instrument such as Micron. Future research will involve planning techniques to lay out more complex patterns that take into account the anatomy of the individual patient. For example, burn placement can be constrained in order to avoid coagulating retinal veins.

While the present study involves stepwise or point-topoint movement of the manipulator for photocoagulation, Micron is equally well-suited to continuous motion to generate smooth trajectories for other laser interventions such as cutting [15]. This is another subject for future research.

\section{ACKNOWLEDGMENT}

Thanks to Mr. Greg Halstead and Mr. Rick Hurst of Iridex Corp. for assistance with the laser.

\section{REFERENCES}

[1] Diabetic Retinopathy Study Research Group, "Photocoagulation treatment of proliferative diabetic retinopathy. Clinical application of Diabetic Retinopathy Study (DRS) findings. DRS report number 8," Ophthalmology, vol. 88, pp. 583-600, 1981.

[2] D. A. Infeld and J. G. O'Shea, "Diabetic retinopathy," Postgrad. Med. J., vol. 74, pp. 129-133, 1998.

[3] F. Bandello, P. Lanzetta, and U. Menchini, "When and how to do a grid laser for diabetic macular edema," Doc. Ophthalmologica, vol. 97, pp. 415-419, 1999.

[4] I. D. Ladas, "Use of digitized fluorescein angiogram system to guide laser treatment of diabetic macular edema: a new technique," Ophthalmologica, vol. 217, pp. 194-198, 2003.

[5] M. S. Blumenkranz, D. Yellachich, D. E. Andersen, M. W. Wiltberger, D. Mordaunt, G. R. Marcellino, and D. Palanker, "Semiautomated pattern scanning laser for retinal photocoagulation," Retina vol. 26, pp. 370-376, 2006.

[6] C. N. Riviere, W. T. Ang, and P. K. Khosla, "Toward active tremor canceling in handheld microsurgical instruments," IEEE Trans. on Robot. and Autom. Proc., vol. 19, pp. 793-800, 2003. 
[7] B. C. Becker, S. Voros, R. A. MacLachlan, G. D. Hager, and C. N. Riviere, "Active guidance of a handheld micromanipulator using visual servoing," in Proc. IEEE Int. Conf. Robot. Autom., Kobe, Japan, 2009.

[8] R. A. MacLachlan, B. C. Becker, and C. N. Riviere, "Control of an active handheld instrument for microsurgery and micromanipulation," in Proc. IEEE Int. Conf. Robot. Autom. Kobe, Japan, 2009.

[9] R. A. MacLachlan and C. N. Riviere, "High-speed microscale optical tracking using digital frequency-domain multiplexing," IEEE Trans. Instrum. Meas., vol. 58, pp. 1991-2001, June 2009.

[10] C. N. Riviere, J. Gangloff, and M. de Mathelin, "Robotic compensation of biological motion to enhance surgical accuracy," Proc. of the IEEE, vol. 94, pp. 1705-1716, 2006.

[11] H. Bay, A. Ess, T. Tuytelaars, and L. van Gool, "SURF: Speeded Up Robust Features," Proc. IEEE CVPR, vol. 110, pp. 346-359, 2008.

[12] M. A. Fischler and R. C. Bolles, "Random sample consensus: A paradigm for model fitting with applications to image analysis and automated cartography," Communications of the ACM, vol. 24, pp. 381-395, 1981

[13] R. Hartley and A. Zisserman, Multiple View Geometry in Computer Vision. Cambridge, UK: Cambridge University Press, 2003.

[14] M.-H. Yang and N. Ahuja, "Gaussian mixture model for human skin color and its applications in image and video databases," Proc. SPIE, vol. 3656, pp. 458-466, 1998.

[15] S. Schastak, Y. Yafai, T. Yasukawa, Y.-S. Wang, G. Hillrichs, and P. Wiedemann, "Flexible UV light guiding system for intraocular laser microsurgery," Lasers in Surgery and Med., vol. 39, pp. 353-357, 2007. 